Tome 49, no 19. - Septembre 1942.

Aus dem Anatomischen Institut der Universität Bern

Direktor: Prof. Dr. H. Bluntschli.

\title{
Vergleichende Beurteilung der Placentation bei den Insektivoren
}

\author{
von

\section{Fritz STRAUSS}

Bern.

Mit 11 Textfiguren und 2 Tabellen.

Auf dem Gebiet der vergleichenden Placentationslehre sind in neuer Zeit viele und wertvolle Arbeiten publiziert worden. Mossman (1937) hat darum in einer monographischen Darstellung versucht, alle bekannten und oft weit zerstreuten Befunde über die Fruchthüllen und die Placenten der Eutherien übersichtlich zu ordnen. Zur Klassifikation der einzelnen Säugetiere auf der Basis des Fruchthüllenvergleiches bediente sich Mossman folgender vier Hauptmerkmale, die er für ordnungsspezifisch hält: 1) der Orientierung der Keimscheibe im Uterus; 2) des Verhaltens und der Grösse des Dottersackes; 3) als sehr wichtiges Charakteristikum der Ausbildung sowie Ausdehnung der entodermalen Allantois, und 4) der feineren Morphologie der definitiven Placenta. Auf dieser Grundlage glaubte er bis dahin unbekannte phylogenetische $\mathrm{Zu}$ sammenhänge zwischen einzelnen Säugerordnungen und Beziehungen unter weit verwandten Familien nachweisen zu können. So konnte er z. B. für die Nagetiere in klarer Beweisführung eine deutliche evolutive Reihe aufzeichnen.

Die damals bekannten Befunde über die Insektivoren gestatteten Mossman aber nicht, Licht in das Dunkel der phylogenetischen Zusammenhänge dieser Gruppe zu bringen. Das Eihautund Placentationsverhalten der Kerfejäger muss aber darum 
besonders interessieren, weil seit HuXLEY (1880) allgemein angenommen wird, dass die Insektenfresser einen zentralen und primitiven Platz unter den Eutheria einnehmen; so dürften sie die nächsten Beziehungen zur Wurzel des Säugetierstammes haben. Aus diesem Bedürfnis heraus haben in den letzten Jahren Goetz (1936, 1937 a u. b) für Centetes und Hemicentetes, Hıll (1939) für Potamogale, Mossman (1939) für Scalopus, Sansom (1937 u. 1939) für Crocidura, S'trauss für Ericulus und Wislocki (1940) für Solenodon die Ergebnisse ihrer Untersuchungen an den Fruchthüllen und Placenten der genannten Insektivorenarten mitgeteilt. Auf Grund der neuen Tatsachen lassen sich nun bei den Insektivoren hinsichtlich des Eihautverhaltens mehrere Gruppen unterscheiden, die bisher, was die vergleichend-embryologischen Verhältnisse anbetrifft, nur wenig Beziehungen zu einander erkennen liessen. Es werden so sich auch mancherlei Umgruppierungen innerhalb der Ordnung als notwendig erweisen. Bei diesen Erwägungen dürfen wir allerdings nicht vergessen, dass die Ordnung der Insektenfresser über 300 rezente Arten umfasst, von denen bisher erst 13 Spezies aus ganz verschiedenen Familien untersucht werden konnten.

Um doch zu einem gewissen Abschluss zu kommen, hat Mossman in seiner Monographie auf Grund der älteren Befunde versucht, die Fruchthüllen der Insektivoren einheitlich $\mathrm{zu}$ charakterisieren: 1) die Keimscheibe soll sich nach ihm im Uterus antimesometral oder orthomesometral (= lateral) orientieren; dabei ist aber streng zwischen Orientierung der Keimscheibe und der Position der ersten Anheftung zu unterscheiden, da das Nidationsverhalten nicht einmal als Gruppen- oder Familiencharakteristikum angesehen wird ; 2) wäre der Dottersack für die ganze Ordnung klein und soll bis zur Geburt bestehen bleiben; ausserdem würden die Kerfejäger prinzipiell zur unvollständigen Keimblätterumkehr neigen; 3) wäre die entodermale Allantois nach diesem Schema mittelgross oder klein, und 4) die definitive Placenta durchwegs labyrinthin-haemochorial. Dass diese hier angedeutete Auffassung nicht in allen Punkten zutreffen kann, möge die folgende Zusammenstellung der bisherigen Ergebnisse beweisen.

Hemicentetes semispinosus (Abb. 1) zeigt nach Gовтz eine superfizielle, intraglanduläre sowie orthomesometrale Implantation, wobei eine Decidua capsularis völlig fehlt. Dieses 
Verhalten ist eine besonders raumschaffende Nidationsart und erklärt sich durch die enorm grosse Fruchtbarkeit der Centetinen (Bluntschli, 1937), die mit Portmann (1938) als ein sehr archaisches Verhalten unter den Säugetieren angesprochen werden darf. Die Keimscheibe orientiert sich ebenfalls lateral. Im weiteren Verlauf der Entwicklung kommt es zur Ausbildung eines grossen Dottersackes, der eine orthomesometral gelegene, sehr hochstehende, aber nur temporäre Choriovitellinplacenta aufbaut. Dieses Placentarorgan verbindet sich mit einem sehr grossen „falschen Placentarkissen“, das in dieser Ausdehnung bisher nur bei den weichhaarigen Borstenigeln bekannt geworden ist.

Derartige Schleimhautkissen, die nicht am Implantationsort auftreten, finden sich nach den bisherigen Erfahrungen dort, wo sie einer Dottersackplacenta als Ernährungsbasis dienen, also bei den Insektivoren nur dann, wenn eine Choriovitellinplacenta als vorübergehendes Stoffwechselorgan funktioniert (Tab. 1).

Die Allantoisblase von Hemicentetes ist gross und bildet die Grundlage für eine am Implantationspol gelegene, weitmaschige Labyrinthplacenta mit einem zentralen Blutbeutel, die den Topfplacenten sehr nahe steht und an die sich peripher ein syndesmochorialer Semiplacentarring anschliesst.

Der ebenfalls auf Madgaskar heimische Borstenigel $C$ e $n$ t e t e $s$ ec a udatus (siehe Goetz), von dem wir Frühstadien nicht mit Sicherheit kennen, zeigt in späteren Phasen der Entwicklung seiner Eihüllen und im Placentarbau, abgesehen von unwesentlichen, kleinen Abweichungen, die selben Verhältnisse wie $\mathrm{He}$ micentetes (Abb. 1).

Der hartstachelige Borstenigel Ericulus setosus (Abb. 2) wurde bislang mit den weichhaarigen Borstenigeln Centetes und Hemicentetes als in die gleiche Insektivoren-Unterfamilie, die Centetinen, gehörend betrachtet. Seine Einbettung scheint nach meinen Untersuchungen antimesometral und teilweise-interstitiellintraglandulär zu erfolgen. Die Keimscheibe liegt antimesometral. In den jüngeren Stadien der Placentarentwicklung kommt es bei ihm ebenfalls zum Aufbau einer physiologisch hochentwickelten, allerdings nur ringförmigen und temporären Choriovitellinplacenta. In der Implantationsachse, gegenüber dem Nidationspol, entwickelt sich eine primitive, zweiblättrige Dottersackplacenta, die hier von einem nur angedeuteten falschen Placentarkissen ernährt wird. Im 
Tab. 1. - Tabellarische Zusammenstellung der wichtigsten Fruchthüllenund Placentationsmerkmale der Insektivoren.

\begin{tabular}{|c|c|c|c|c|c|c|}
\hline $\begin{array}{l}\text { Fa- } \\
\text { milie }\end{array}$ & Art & $\begin{array}{c}\text { Implan- } \\
\text { tation }\end{array}$ & $\begin{array}{l}\text { Lage der } \\
1 . \text { An- } \\
\text { heftung }\end{array}$ & $\begin{array}{l}\text { Orientie- } \\
\text { rung der } \\
\text { Keim- } \\
\text { scheibe }\end{array}$ & $\begin{array}{l}\text { Decidua } \\
\text { capsu- } \\
\text { laris }\end{array}$ & Dottersack \\
\hline \multirow{3}{*}{$\begin{array}{l}\text { Cente- } \\
\text { tinae }\end{array}$} & $\begin{array}{l}\text { Centetes } \\
\text { ecaudatus }\end{array}$ & $\begin{array}{c}\text { zentral- } \\
\text { intra- } \\
\text { glandulär }\end{array}$ & $\begin{array}{l}\text { ortho- } \\
\text { meso- } \\
\text { metral }\end{array}$ & $\begin{array}{l}\text { ortho- } \\
\text { meso- } \\
\text { metral }\end{array}$ & fehlt & $\begin{array}{l}\text { gross; temporäre } \\
\text { Ch.-V.-Placenta }\end{array}$ \\
\hline & $\begin{array}{l}\text { Hemicente- } \\
\text { tes semi- } \\
\text { spinosus }\end{array}$ & $\begin{array}{l}\text { zentral- } \\
\text { intra- } \\
\text { glandulär }\end{array}$ & $\begin{array}{l}\text { ortho- } \\
\text { meso- } \\
\text { metral }\end{array}$ & $\begin{array}{l}\text { ortho- } \\
\text { meso- } \\
\text { metral }\end{array}$ & fehlt & $\begin{array}{l}\text { gross; temporäre } \\
\text { Ch.-V.-Placenta }\end{array}$ \\
\hline & $\begin{array}{l}\text { Ericulus } \\
\text { setosus }\end{array}$ & $\begin{array}{c}\text { exzen- } \\
\text { trisch- } \\
\text { intra- } \\
\text { glandulär } \\
\end{array}$ & $\begin{array}{l}\text { anti- } \\
\text { meso- } \\
\text { metral }\end{array}$ & $\begin{array}{l}\text { anti- } \\
\text { meso- } \\
\text { metral }\end{array}$ & $\begin{array}{l}\text { ange- } \\
\text { deutet }\end{array}$ & $\begin{array}{l}\text { gross; bilaminäre } \\
\text { D.-S.-Placenta; tem- } \\
\text { por. Ch.-V.-Placenta; } \\
\text { inkompl. Inversion }\end{array}$ \\
\hline $\begin{array}{c}\text { Pota- } \\
\text { moga- } \\
\text { lidae }\end{array}$ & $\begin{array}{c}\text { Potamogale } \\
\text { velox }\end{array}$ & $?$ & $?$ & $?$ & $?$ & klein \\
\hline $\begin{array}{l}\text { Chry-- } \\
\text { sochlo- } \\
\text { ridae }\end{array}$ & $\begin{array}{l}\text { Chryso- } \\
\text { chloris } \\
\text { aurea }\end{array}$ & zentral & $\begin{array}{l}\text { ortho- } \\
\text { meso- } \\
\text { metral }\end{array}$ & $\begin{array}{l}\text { meso- } \\
\text { metral }\end{array}$ & fehlt & $\begin{array}{l}\text { gross; temporäre } \\
\text { Ch.-V.-Placenta; } \\
\text { inkompl. Inversion }\end{array}$ \\
\hline \multirow{2}{*}{$\begin{array}{l}\text { Erina- } \\
\text { ceidae }\end{array}$} & $\begin{array}{l}\text { Erinaceus } \\
\text { europaeus }\end{array}$ & $\begin{array}{l}\text { intersti- } \\
\text { tiell }\end{array}$ & $\begin{array}{l}\text { anti- } \\
\text { meso- } \\
\text { metral }\end{array}$ & $\begin{array}{l}\text { anti- } \\
\text { meso- } \\
\text { metral }\end{array}$ & $\begin{array}{l}\text { kom- } \\
\text { plett }\end{array}$ & $\begin{array}{l}\text { mittelgross; persist. } \\
\text { Ch.-V.-Placenta; } \\
\text { inkompl. Inversion }\end{array}$ \\
\hline & Gymnura & $?$ & $\begin{array}{l}\text { anti- } \\
\text { meso- } \\
\text { metral }\end{array}$ & $\begin{array}{l}\text { anti- } \\
\text { meso- } \\
\text { metral }\end{array}$ & $?$ & $?$ \\
\hline \multirow{3}{*}{$\begin{array}{l}\text { Sori- } \\
\text { cidae }\end{array}$} & $\begin{array}{c}\text { Sorex } \\
\text { vulgaris }\end{array}$ & zentral & bilateral & $\begin{array}{l}\text { anti- } \\
\text { meso- } \\
\text { metral }\end{array}$ & $\begin{array}{l}\text { ange- } \\
\text { deutet }\end{array}$ & $\begin{array}{l}\text { gross; persistente } \\
\text { Ch.-V.-Placenta; } \\
\text { inkompl. Inversion }\end{array}$ \\
\hline & $\begin{array}{l}\text { Crocidura } \\
\text { caerulea }\end{array}$ & $\begin{array}{l}\text { exzen- } \\
\text { trisch }\end{array}$ & $\begin{array}{l}\text { anti- } \\
\text { meso- } \\
\text { metral }\end{array}$ & $\begin{array}{l}\text { anti- } \\
\text { meso- } \\
\text { metral }\end{array}$ & $\begin{array}{l}\text { kom- } \\
\text { plett }\end{array}$ & $\begin{array}{l}\text { gross; persistente } \\
\text { Ch.-V.-Placenta; } \\
\text { inkompl. Inversion }\end{array}$ \\
\hline & $\begin{array}{l}\text { Solenodon } \\
\text { paradoxus }\end{array}$ & $\begin{array}{l}\text { exzen- } \\
\text { trisch ? } \\
\text { inter- } \\
\text { stitiell? }\end{array}$ & $\begin{array}{l}\text { anti- } \\
\text { meso- } \\
\text { metral }\end{array}$ & $?$ & $\begin{array}{l}\text { ange- } \\
\text { deutet }\end{array}$ & $\begin{array}{l}\text { gross; persistente } \\
\text { Ch.-V.-Placenta; } \\
\text { komplete Inversion }\end{array}$ \\
\hline \multirow[t]{2}{*}{$\begin{array}{c}\text { Talpi- } \\
\text { dae }\end{array}$} & $\begin{array}{c}\text { Talpa } \\
\text { europaea }\end{array}$ & zentral & $\begin{array}{l}\text { bilateral } \\
\text { bis anti- } \\
\text { meso- } \\
\text { metral }\end{array}$ & $\begin{array}{l}\text { anti- } \\
\text { meso- } \\
\text { metral }\end{array}$ & $\begin{array}{l}\text { ange- } \\
\text { deutet }\end{array}$ & $\begin{array}{l}\text { klein; bilaminäre } \\
\text { D.-S.-Placenta; } \\
\text { persistente Ch.-V.- } \\
\text { Placenta; } \\
\text { inkompl. Inversion }\end{array}$ \\
\hline & $\begin{array}{l}\text { Scalopus } \\
\text { aquaticus }\end{array}$ & $?$ & $?$ & $?$ & $?$ & $\begin{array}{l}\text { klein; persistente } \\
\text { Ch.-V.-Placenta }\end{array}$ \\
\hline $\begin{array}{c}\text { Tupai- } \\
\text { idae }\end{array}$ & $\begin{array}{c}\text { Tupaja } \\
\text { javanica }\end{array}$ & zentral & bilateral & $\begin{array}{l}\text { anti- } \\
\text { meso- } \\
\text { metral }\end{array}$ & fehlt & $\begin{array}{l}\text { klein; temporäre } \\
\text { Ch.-V.-Placenta }\end{array}$ \\
\hline
\end{tabular}


Tab. 1. - Tabellarische Zusammenstellung der wichtigsten Fruchthüllenund Placentationsmerkmale der Insektivoren.

\begin{tabular}{|c|c|c|c|c|c|}
\hline Art & $\begin{array}{l}\text { Falsches } \\
\text { Placentar- } \\
\text { kissen }\end{array}$ & $\begin{array}{l}\text { Entoder- } \\
\text { males } \\
\text { Allantois }\end{array}$ & Definitive Placenta & $\begin{array}{l}\text { Akzesso- } \\
\text { rische } \\
\text { Placenten }\end{array}$ & Autor \\
\hline $\begin{array}{l}\text { Centetes } \\
\text { ecaudatus }\end{array}$ & $\begin{array}{l}\text { sehr gross; } \\
\text { orthomeso- } \\
\text { metral }\end{array}$ & gross & $\begin{array}{c}\text { discoidale, } \\
\text { haemochoriale } \\
\text { All.-Ch.-Placenta } \\
\text { (Übergangsform) }\end{array}$ & $\begin{array}{c}\text { zentraler } \\
\text { Blutbeutel; } \\
\text { Semipla- } \\
\text { centarring } \\
\end{array}$ & $\begin{array}{l}\text { GOETZ } \\
(1936)\end{array}$ \\
\hline $\begin{array}{l}\text { Hemicentetes } \\
\text { semispinosus }\end{array}$ & $\begin{array}{l}\text { sehr gross; } \\
\text { orthomeso- } \\
\text { metral }\end{array}$ & gross & $\begin{array}{l}\text { discoidale, } \\
\text { haemochoriale } \\
\text { All.-Ch.-Placenta } \\
\text { (Übergangsform) }\end{array}$ & $\begin{array}{c}\text { zentraler } \\
\text { Blutbeutel; } \\
\text { Semipla- } \\
\text { centarring } \\
\end{array}$ & $\begin{array}{c}\text { GOETZ } \\
(1937 a \text { u. b) }\end{array}$ \\
\hline $\begin{array}{l}\text { Ericulus } \\
\text { setosus }\end{array}$ & $\begin{array}{c}\text { klein; } \\
\text { mesometral }\end{array}$ & gross & $\begin{array}{c}\text { discoidale, } \\
\text { haemochoriale, } \\
\text { labyrinthine } \\
\text { All.-Ch.-Placenta }\end{array}$ & $\begin{array}{c}\text { zentraler } \\
\text { Blutbeutel }\end{array}$ & Strauss \\
\hline $\begin{array}{l}\text { Potamogale } \\
\text { velox }\end{array}$ & $?$ & gross & $\begin{array}{c}\text { discoidale, } \\
\text { haemochoriale } \\
\text { labyrinthine } \\
\text { All.-Ch.-Placenta }\end{array}$ & $\begin{array}{l}\text { diffuse } \\
\text { Placenta }\end{array}$ & $\begin{array}{l}\text { HILL } \\
(1939)\end{array}$ \\
\hline $\begin{array}{c}\text { Chrysochloris } \\
\text { aurea }\end{array}$ & $\begin{array}{c}\text { klein; } \\
\text { orthomeso- } \\
\text { metral }\end{array}$ & mittelgross & $\begin{array}{c}\text { discoidale, } \\
\text { haemochoriale, } \\
\text { labyrinthine } \\
\text { All.-Ch.-Placenta }\end{array}$ & & $\begin{array}{c}\text { DE LANGE } \\
(1919)\end{array}$ \\
\hline $\begin{array}{l}\text { Erinaceus } \\
\text { europaeus }\end{array}$ & & mittelgross & $\begin{array}{c}\text { discoidale, } \\
\text { haemochoriale, } \\
\text { labyrinthine } \\
\text { All.-Ch.-Placenta }\end{array}$ & & $\begin{array}{c}\text { HUBRECHT } \\
(1889) \\
\text { RESINK } \\
(1903)\end{array}$ \\
\hline Gymnura & & $?$ & $?$ & & $\begin{array}{l}\text { HUB RECHT } \\
(1898)\end{array}$ \\
\hline $\begin{array}{l}\text { Sorex } \\
\text { vulgaris }\end{array}$ & $\begin{array}{l}\text { klein; } \\
\text { ringförmig }\end{array}$ & klein & $\begin{array}{c}\text { discoidale, } \\
\text { haemochoriale, } \\
\text { labyrinthine } \\
\text { All.-Ch.-Placenta }\end{array}$ & & $\begin{array}{l}\text { HUBRECHT } \\
(1893)\end{array}$ \\
\hline $\begin{array}{l}\text { Crocidura } \\
\text { caerulea }\end{array}$ & & sehr klein & $\begin{array}{c}\text { discoidale, } \\
\text { haemochoriale, } \\
\text { labyrinthine } \\
\text { All.-Ch.-Placenta }\end{array}$ & $\begin{array}{l}\text { Tropho- } \\
\text { blast- } \\
\text { scheide }\end{array}$ & $\begin{array}{c}\text { SANSOM } \\
(1937,1939)\end{array}$ \\
\hline $\begin{array}{l}\text { Solenodon } \\
\text { paradoxus }\end{array}$ & & fehlt (?) & $\begin{array}{c}\text { discoidale, } \\
\text { haemochoriale, } \\
\text { labyrinthine } \\
\text { All.-Ch.-Placenta }\end{array}$ & $\begin{array}{l}\text { Tropho- } \\
\text { blast- } \\
\text { kissen }\end{array}$ & $\begin{array}{l}\text { WISLOCKI } \\
(1940)\end{array}$ \\
\hline $\begin{array}{c}\text { Talpa } \\
\text { europaea }\end{array}$ & $\begin{array}{l}\text { klein; } \\
\text { ringförmig }\end{array}$ & $\begin{array}{c}\text { mittelgross } \\
\text { bis gross }\end{array}$ & $\begin{array}{c}\text { discoidale, } \\
\text { haemochoriale, } \\
\text { labyrinthine } \\
\text { All.-Ch.-Placenta }\end{array}$ & & $\begin{array}{c}\text { STRAHL } \\
(1892) \\
\text { VERNHOUT } \\
(1895) \\
\end{array}$ \\
\hline $\begin{array}{l}\text { Scalopus } \\
\text { aquaticus }\end{array}$ & $?$ & $\begin{array}{c}\text { mittelgross } \\
\text { bis gross }\end{array}$ & $\begin{array}{l}\text { diffuse, } \\
\text { epitheliochoriale, } \\
\text { labyrinthine } \\
\text { All.-Ch.-Placenta }\end{array}$ & & $\begin{array}{c}\text { Mossman } \\
(1939)\end{array}$ \\
\hline $\begin{array}{c}\text { Tupaja } \\
\text { javanica }\end{array}$ & $\begin{array}{l}\text { mittelgross, } \\
\text { später echte } \\
\text { Kissen }\end{array}$ & $\begin{array}{c}\text { mittelgross } \\
\text { bis gross }\end{array}$ & $\begin{array}{c}\text { bidiscoidale, } \\
\text { haemochoriale, } \\
\text { labyrinthine } \\
\text { All.-Ch.-Placenta }\end{array}$ & & $\begin{array}{l}\text { DE LANGE } \\
\& \text { NIER- } \\
\text { STRASZ } \\
(1932)\end{array}$ \\
\hline
\end{tabular}


Vergleich zu Centetes und Hemicentetes zeigt Ericulus eine stärkere Umlsehr seiner Fruchthüllen, da bei ihm der Dottersack noch mehr eingedellt wird. Am Placentarpol selbst entwickelt sich eine engmaschige Labyrinthplacenta mit einem zentralen, stark gefalteten Blutbeutel. Weitere Nebenplacenten fehlen.

Die Wasserspitzmaus Potamogale velox (siehe Hill) (Abb. 3), die nach der bisher gültigen Auffassung ebenfalls zu den Centetiden gezählt wird, scheint auf Grund ihres Fruchthüllenverhaltens in den allein bekannt gewordenen Spätstadien Centetes und Hemicentetes nicht fern zu stehen. Die Placentation ist vermutlich rein allantoid. Die entodermale Allantois ist gross und wird durch die Nabelschnurgefässe unvollständig in vier Kammern geteilt. Am Placentarpol findet sich eine kleine, haemochoriale Labyrinthplacenta. An sie schliesst eine epitheliochoriale Placenta von grosser Ausdehnung an, die dem Rest der Decidua überall anliegt. Der Dottersack scheint (nach den von Hill bisher mitgeteilten Untersuchungsergebnissen) klein zu sein.

Der Goldmaulwurf Chrysochloris a urea (siehe DE LANGE) (Abb. 4) zeigt bei lateraler und superfizieller Implantation eine mesometrale Orientierung der Keimscheibe. Allerdings bezeichnet Mossman ihre Lage als eine laterale Modifikation des antimesometralen Typus; aber sowohl seine wie DE LANGes Abbildungen sprechen viel mehr für eine mesometrale Orientierung. Ebenso findet sich später in orthomesometraler Stellung eine kappenförmige und vorübergehende Choriovitellinplacenta mit einem ernährenden falschen Placentarkissen. In den ältesten, bisher untersuchten Placentarstadien von Chrysochloris ist der Dottersack praktisch ganz verschwunden (nach brieflicher Mitteilung von de Lange an Hintzsche, 1940) und die gestielte Placenta wird, im Vergleich zu den Centetinen, nur von einem bescheidenen Allantoisrest umgeben. Chrysochloris zeigt ausserdem eine leichte Inversion der Eihäute.

Die Placenta und die Fruchthüllen des europäischen Igels Erinaceus europaeus (Abb. 5) sind durch die grundlegenden Arbeiten Hubrechts und seines Schülers Resink allgemein bekannt geworden. Die Keimscheibe ist in utero antimesometral orientiert und wird relativ bald von einer vollständigen Decidua capsularis umschlossen. In den jüngeren Placentarphasen findet sich ein mittelgrosser Dottersack, der später teilweise 
invertiert wird. Anfänglich ist auch eine ringförmige Choriovitellinplacenta vorhanden, die trotz starker Schrumpfung bis zur Geburt nachweisbar bleibt. Der haemochorialen Labyrinthplacenta liegt eine nur mittelgrosse Allantois an.

Zwischen Erinaceus und den Soriciden besteht auf Grund der Fruchthüllen möglicherweise eine nahe phylogenetische Beziehung, denn bei der Waldspitzmaus Sorex oulgaris (siehe Hubrecht) (Abb. 6) wird der relativ grosse Dottersack stärker invertiert als bei den Erinaceiden. Der embryonale Harnsack scheint verhältnismässig klein zu sein. Der Keim implantiert sich primär bilateral und seine Scheibe ist antimesometral orientiert. Zufolge der zentralen Einbettung kann eine kapsuläre Decidua nur angedeutet vorhanden sein. Entsprechend der bilateralen Implantation wird mit Hilfe einer ringförmigen, kissenartigen Schleimhautanschwellung eine temporäre, gürtelförmige Choriovitallinplacenta aufgebaut.

Die indische Spitzmaus Crocidura caer u le a (siehe Sansom) (Abb. 7) steht den Soriciden auch vom vergleichend-embryologischen Standpunkt aus recht nahe. Die Implantation dürfte teilweise-interstitiell und antimesometral erfolgen; auch die Keimscheibe liegt antimesometral. Anfangs ist eine komplette Decidua capsularis vorhanden, die aber mit zunehmender Ausdehnung des Keimes allmählich verschwindet. Der Dottersack ist gross und wird in den ältesten Entwicklungsstadien weitgehend invertiert, sodass nur noch ein spaltförmiges Lumen vorhanden ist. Die entodermale Allantois (in meinem Schema gar nicht dargestellt) ist sehr klein und überhaupt nur kurze Zeit als Bläschen nachweisbar. Es kommt früh zur Ausbildung einer ringförmigen Dottersackplacenta, die bis zum Wurf erhalten bleibt. Die definitive Labyrinthplacenta ist allanto-haemochorial. Die allantoiden Nabelschnurgefässe durchsetzen, ehe sie an die Placenta gelangen, eine dichte Trophoblastscheide, die nach den Feststellungen von SAnsom, etwa in ähnlicher Weise wie der Blutbeutel von Ericulus, im Dienst der Nahrungsaufbereitung für den Fetus stehen dürfte.

Der auf San Domingo heimische Solenodon paradoxus (siehe Wislock I) (Abb. 8), von dem erst ein trächtiger Fruchthalter untersucht werden konnte, schliesst sich gerade in bezug auf die Neigung zur Keimblätterumkehr den Soricoidae leicht an, obwohl er auf Grund der bisher gebräuchlichen, taxonomischen Merkmale 
zu den Centetoidae gezählt wurde. Er zeigt eine komplette Inversion seiner Fruchthüllen. Eine entodermale Allantois fehlt wahrscheinlich völlig; deren Bildung scheint überhaupt mit fortschreitender Keimblätterumkehr schrittweise zu unterbleiben. Da bisher nur eine ältere Placentarphase von Solenodon beschrieben werden konnte, fehlen noch Angaben über den Implantationsmodus; allerdings waren in dem untersuchten Fall Reste einer Decidua capsularis vorhanden. Neben der allantoiden, haemochorialen Placenta persistiert eine primäre Choriovitellinplacenta offenbar bis zur Geburt. In gleicher Weise wie bei Crocidura liegt auch hier über dem haemochorialen Placentarlabyrinth ein dickes Trophoblastpolster. Diese nicht fetal vaskularisierte Zone besteht aus anastomosierenden Cytotrophoblastzellhaufen, die untereinander zusammenhängende Spalträume umschliessen. Diese Spalten stehen mit den mütterlichen Blutlakunen in Verbindung, enthalten aber wenig Blut. Eine gewisse Ähnlichkeit dieses Trophoblastpolsters mit dem stark gefalteten Blutbeutel von Ericulus ist auch hier nicht zu verkennen.

Der europäische Maulwurf Tal pa europaea (siehe Strahl, Vernhout) (Abb. 9) implantiert sich superfiziell und hat darum auch nur eine angedeutete Decidua capsularis. Die Nidation erfolgt entweder bilateral oder antimesometral, während die Keimscheibe stets antimesometral orientiert ist. Neben einem kleinen Dottersack mit unvollständiger Keimblätterumkehr findet sich eine mittelgrosse Allantoisblase. Einer gürtelförmigen temporären Choriovitellinplacenta dient ein kleines, ringförmiges falsches Placentarkissen als Ernährungsgrundlage. Die definitive Placenta ist nach den Untersuchungen von Vernhout als allantoid-haemochorial anzusprechen, während Grosser (1927 u. 1935) die Auffassung vertritt, die Talpaplacenta sei in die endotheliochoriale Gruppe einzureihen.

Völlig anders als Talpa verhält sich in seinem späteren Placentarbau der amerikanische Maulwurf Scalopus aquaticus (siehe Mossman) (Abb. 10). Hier findet sich eine rein epitheliochoriale Placenta mit leicht angedeuteten Zotten. Auch sind die für die Halbplacenten charakteristischen Chorionblasen vorhanden. Diese Semiplacenta lässt nur eine kleine mesometrale Kalotte an der Fruchtblase frei. Bis zur Funktionsaufnahme der diffusen Placenta arbeitet eine vorübergehende Dottersackplacenta als kindliches 
Stoffwechselorgan, das aus einer kleinen Nabelblase hervorgegangen ist. Die entodermale Allantois soll wie beim europäischen Maulwurf mittelgross sein.

Die Einordnung von T и paja javanica (siehe DE LANGE und Nierstrasz) (Abb. 11) bei den Insektivoren ist schon mehrfach bestritten worden; auch die bisher bekannt gewordenen embryologischen Tatsachen lassen erhebliche Zweifel an der Richtigkeit dieser Klassifizierung auftauchen. Die Implantation des TupajaKeimes erfolgt bilateral und superfiziell; eine kapsuläre Decidua fehlt. Die Keimscheibe ist antimesometral orientiert. Der Dottersack ist klein und die temporäre Choriovitellinplacenta liegt zwei ringförmigen falschen Placentarkissen an, die nach Ausbildung der doppelscheibenförmigen, allanto-haemochorialen Labyrinthplacenta zu echten Placentarkissen werden. Die Allantoisblase selbst ist mittelgross.

Tabelle 2 versucht nun auf Grund der zitierten Arbeiten in einer tabellarischen Übersicht die eingangs genannten vier Hauptmerkmale für die 13 bisher untersuchten Insektivorenarten darzustellen. Die Orientierung der Keimscheibe im Uterus kann darnach als ziemlich einheitlich bezeichnet werden. Bei den beiden weichhaarigen Centetinen sieht sie nach lateral und bei den Arten mit Neigung zur Keimblätterumkehr, Ericulus bis Tupaja (ohne Chrysochloris), scheint nach den bisherigen Ergebnissen die antimesometrale Lage vorzuherrschen. Die orthomesometrale Stellung bei den weichhaarigen Borstenigeln erklärt sich vermutlich durch die grosse Fruchtbarkeit dieser Tiere. Darum ist vielleicht auch der Schluss gestattet, es sei die antimesometrale Lage der Keimscheibe im Uterus als die evolutierte Modifikation eines ursprünglich orthomesometralen Typus anzusehen. Die mesometrale Orientierung der Keimscheibe bei Chrysochloris ist, wie auch Mossman hervorhebt, noch nicht genügend bewiesen, sodass das abweichende Verhalten des Goldmaulwurfes event. später nicht bestätigt werden dürfte. Die Position des Implantationsortes fällt, abgesehen von Chrysochloris und den drei bilateral sich implantierenden Formen (Sorex, Crocidura und Tupaja), stets mit der Orientierungsrichtung der Keimscheibe zusammen.

Das Verhalten des Dottersackes ist dagegen nicht einheitlich: er ist bei der Mehrzahl der bisher untersuchten Insektivorenformen (im Gegensatz zu den Angaben von Mossman) gross und nur bei 
Tab. 2. - Tabellarische Übersicht der vier charakteristischen Eihautmerkmale für die bisher untersuchten Insektivoren.

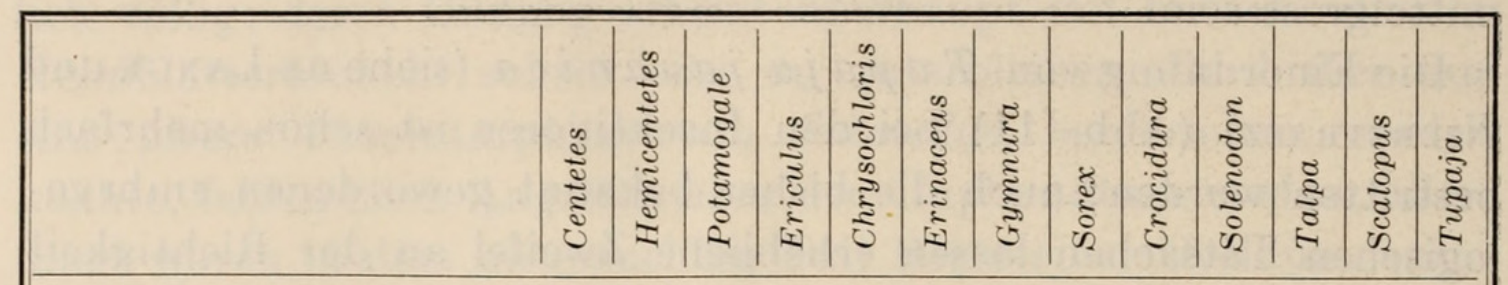

Orientierung der Keimscheibe:

mesometral.

orthomesometral

antimesometral .

Dottersack :

gross :

permanent . . . . . .

temporär

klein :

permanent . . . . .

temporär

Inversion :

inkomplett . . . . . .

komplett
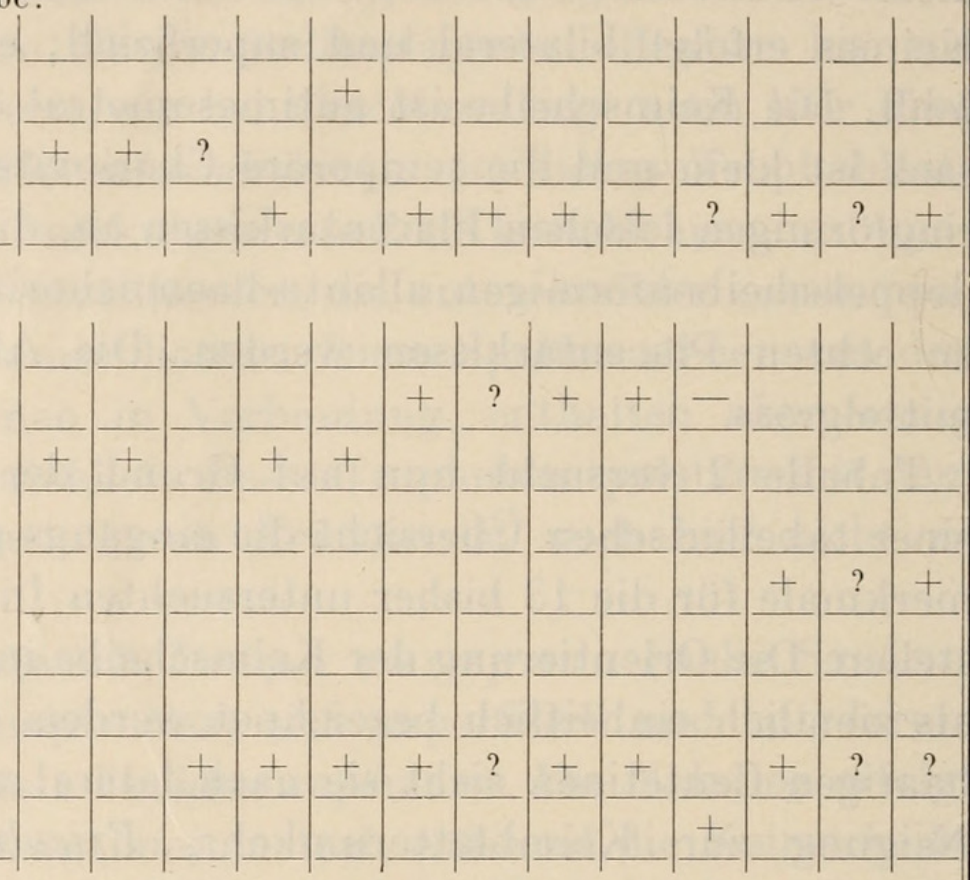

Allantoisblase :

gross

mittelgross

klein

Morphologie der Placenta:

villös, epitheliochorial .

villös, syndesmochorial

labyr., endotheliochorial

labyr., haemochorial

trans., haemochorial

villös, haemochorial . . .
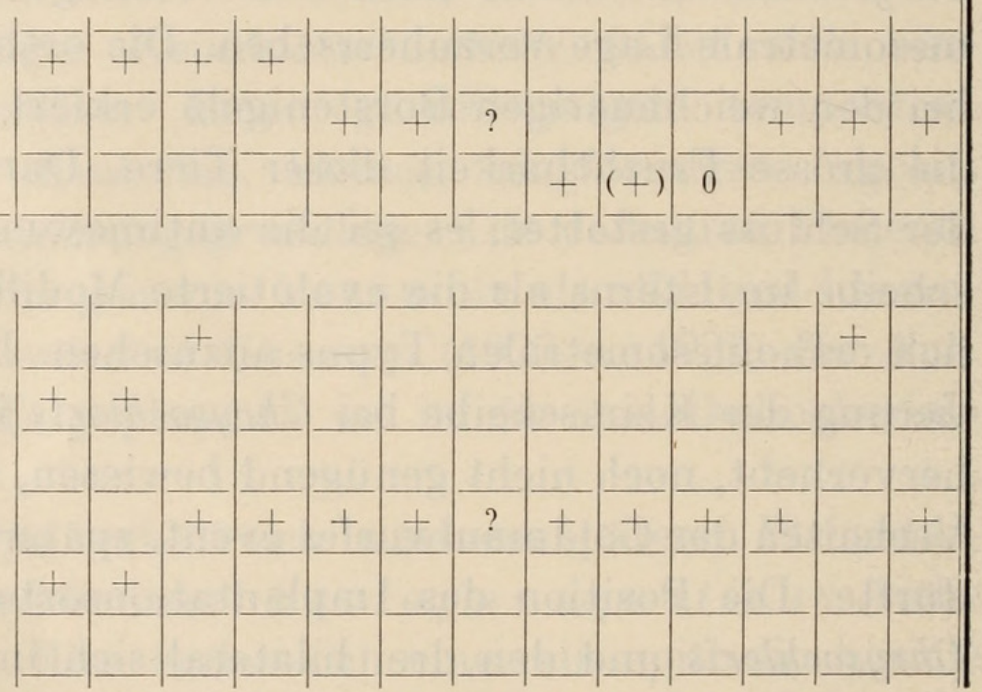

Potamogale, Talpa, Scalopus (?) und Tupaja klein. Bei den Arten mit Inversion der Fruchthüllen muss natürlich die Nabelblase in irgendeiner Form persistieren, denn sonst ist eine bis zur Geburt funktionstüchtige Splanchnopleura nicht vorstellbar. Mit Ausnahme 
der beiden weichhaarigen Borstenigel Centetes und Hemicentetes findet sich von Ericulus über Chrysochloris, Erinaceus und die Soriciden zu Solenodon ein fliessender Übergang der Keimblätterumkehr bis zur völligen Inversion. Damit geht, wie schon angedeutet, eine Grössenabnahme der entodermalen Allantois bis zum völligen Verschwinden parallel. Der europäische und der amerikanische Maulwurf, Talpa und Scalopus, haben ebenso wie Tupaja neben einem kleinen Dottersack eine mittelgrosse Allantois. Sie neigen wohl ebenfalls zur Keimblätterumkehr, wobei sich diese Vermutung auf die Grösse der Allantoisblasen stützt. Aus den Untersuchungen von Hintzsche wissen wir, dass, im Gegensatz zu einer weit verbreiteten Ansicht, zwischen der Grösse der entodermalen Allantois und der Urnierenfunktion bei den centetoiden Kerfejägern keine sicheren Beziehungen bestehen. So kann möglicherweise in dem reziproken Verhältnis von Keimblätterumkehr zur Allantoisausbildung eine gegenseitige Abhängigkeit physiologischer Prozesse ihren Ausdruck finden.

Zur feineren Morphologie der definitiven Placenta lässt sich sagen, dass wobl die labyrinthine, hämochoriale Form vorherrscht; aber es kommen sowohl, wie Centetes und Hemicentetes zeigen, Übergänge zur Topfplacenta als auch zu Semiplacenten, wie sie die gleichen Spezies und Potamogale zeigen, vor. Centetes und Hemicentetes haben einen syndesmochorialen Semiplacentarring, während Potamogale neben seiner zentralen Labyrinthplacenta eine grosse epitheliochoriale Placenta besitzt. Zum Unterschied aller bisher untersuchten Insektivoren zählt Scalopus zu den Adeciduaten, denn er hat eine ausschliesslich epitheliochoriale Placenta. So zeigt sich auch hier wieder die grosse Mannigfaltigkeit im Aufbau der Insektivorenplacenta, die die Schlüsselstellung der Insektenfresser von neuem bekräftigt. Die weichhaarigen Borstenigel verdienen dabei eine besondere Beachtung, weil sie unter den Kerfejägern einer hypothetischen Carnivoren-Insektivorenwurzel des Säugetierstammbaumes am nächsten stehen dürften. Auch die Beziehungen dieser Centetinen zu den Ungulaten und Lemuriden sind in phylogenetischer Hinsicht von Bedeutung, worauf schon Goetz hingewiesen hat. Potamogale und Scalopus, welcher bisher zu den Talpiden gezählt wird, könnten sich vielleicht zukünftig auf Grund ihrer Fruchthüllen- und Placentarverhältnisse als Bindeglieder zwischen den madagassischen Borstenigeln und den Halbaffen 


\section{ERKLÄRUNG DER FIGUREN}

Schematische Darstellung der Fruchthüllenbildung und Placentation einzelner Insektivoren (modifiziert und vervollständigt nach Mossma $\mathrm{N}$ ). In Figur 1 sind die Verhältnisse für Hemicentetes dargestellt, doch gilt dabei die älteste Phase infolge weitgehender Ähnlichkeit auch für Centetes.
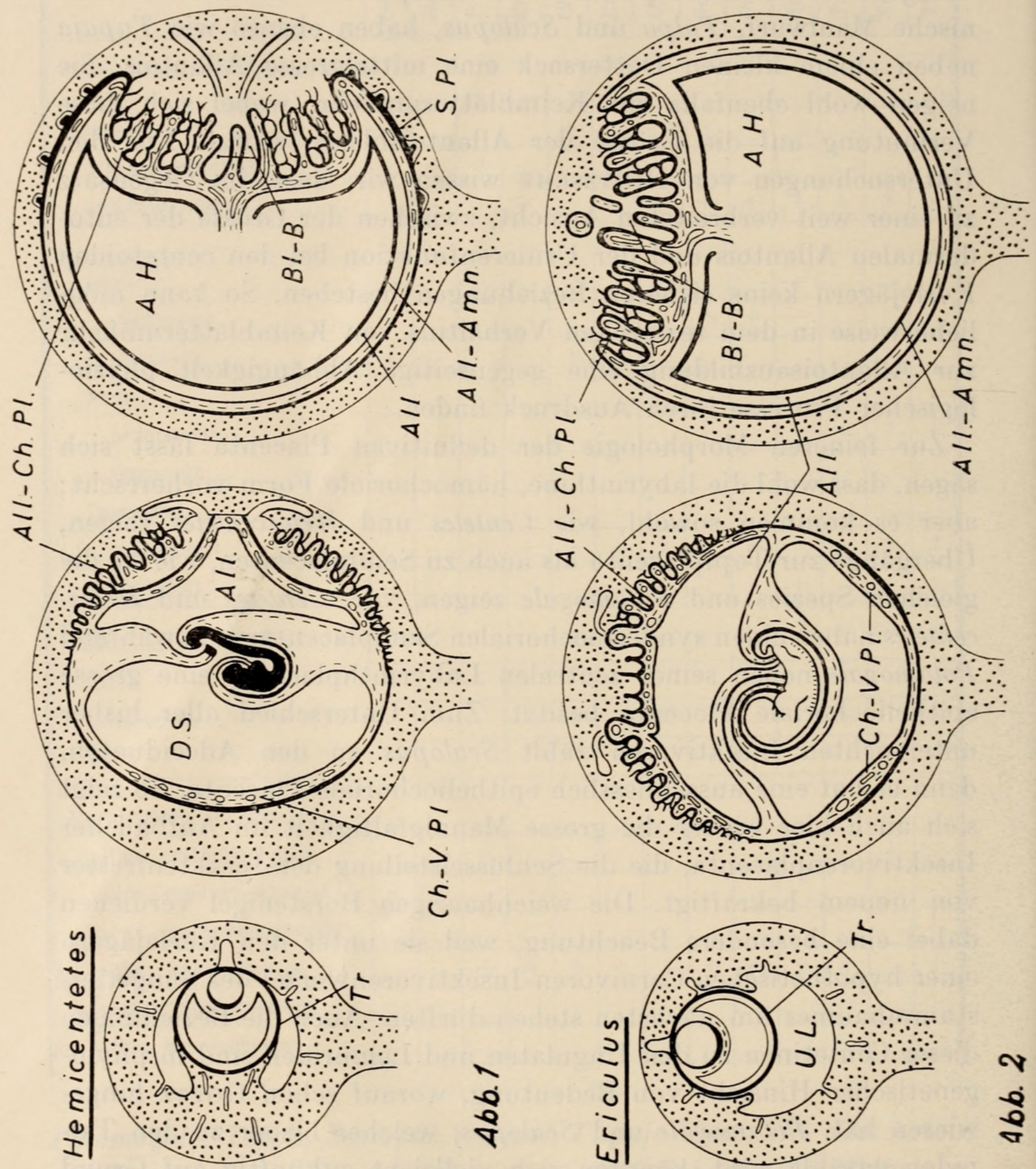
A b k ür z u n g en : A.H. = Amnionhöhle, All. = entodermale Allantois, All.-Amn. = Allantoamnion, All.-Ch.Pl. = Allantochorionplacenta, Bl.-B. = Blutbeutel, Ch.-V.Pl. = Choriovitellinplacenta, D.C. = Decidua capsularis, D.S. $=$ Dottersack, E.C. $=$ Exocoelom, S.Pl. $=$ Semiplacenta, Tr. = Trophoblast, U.L. = Uteruslumen, Z.spl. = splanchnopleurale Zotten.
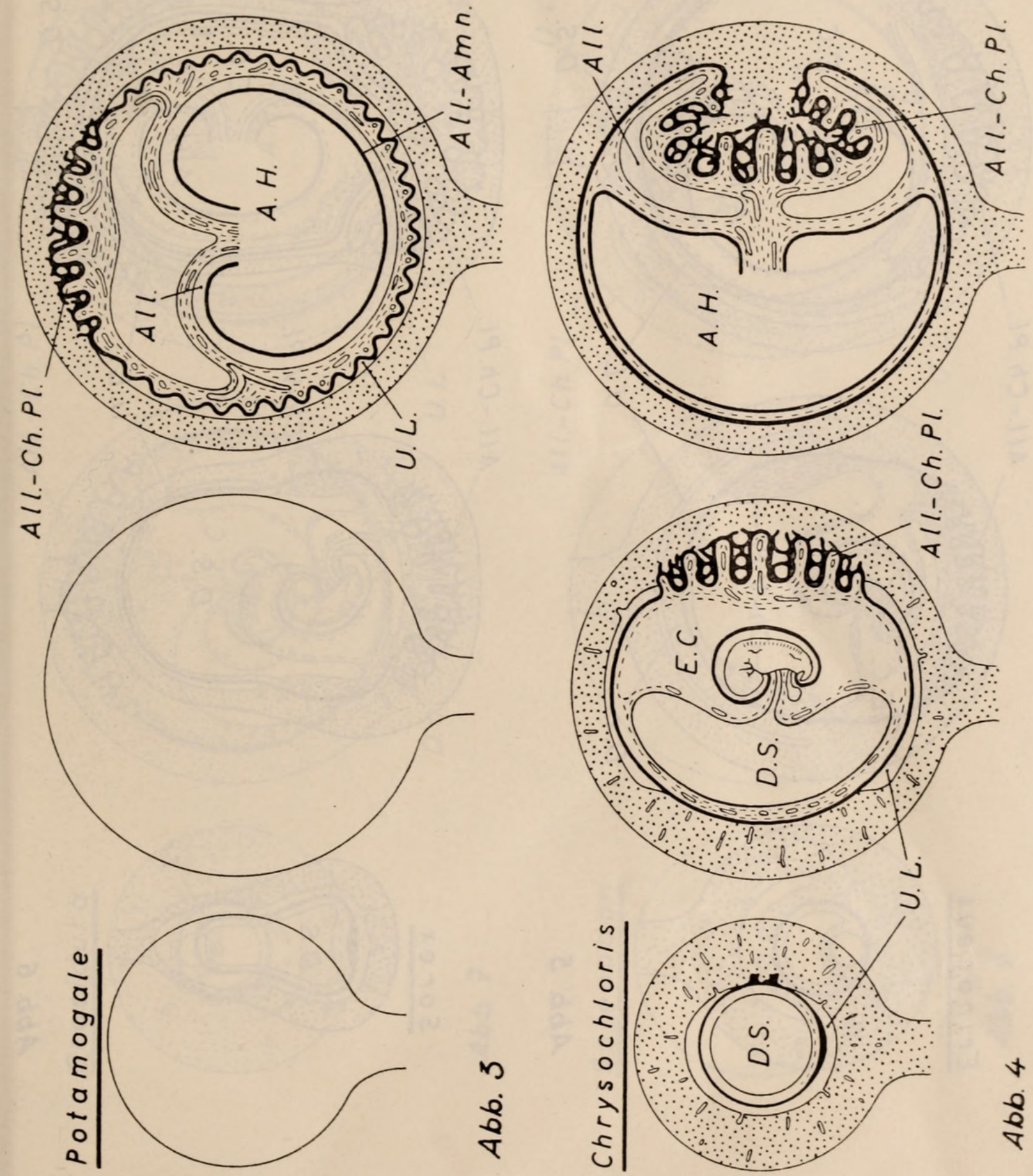

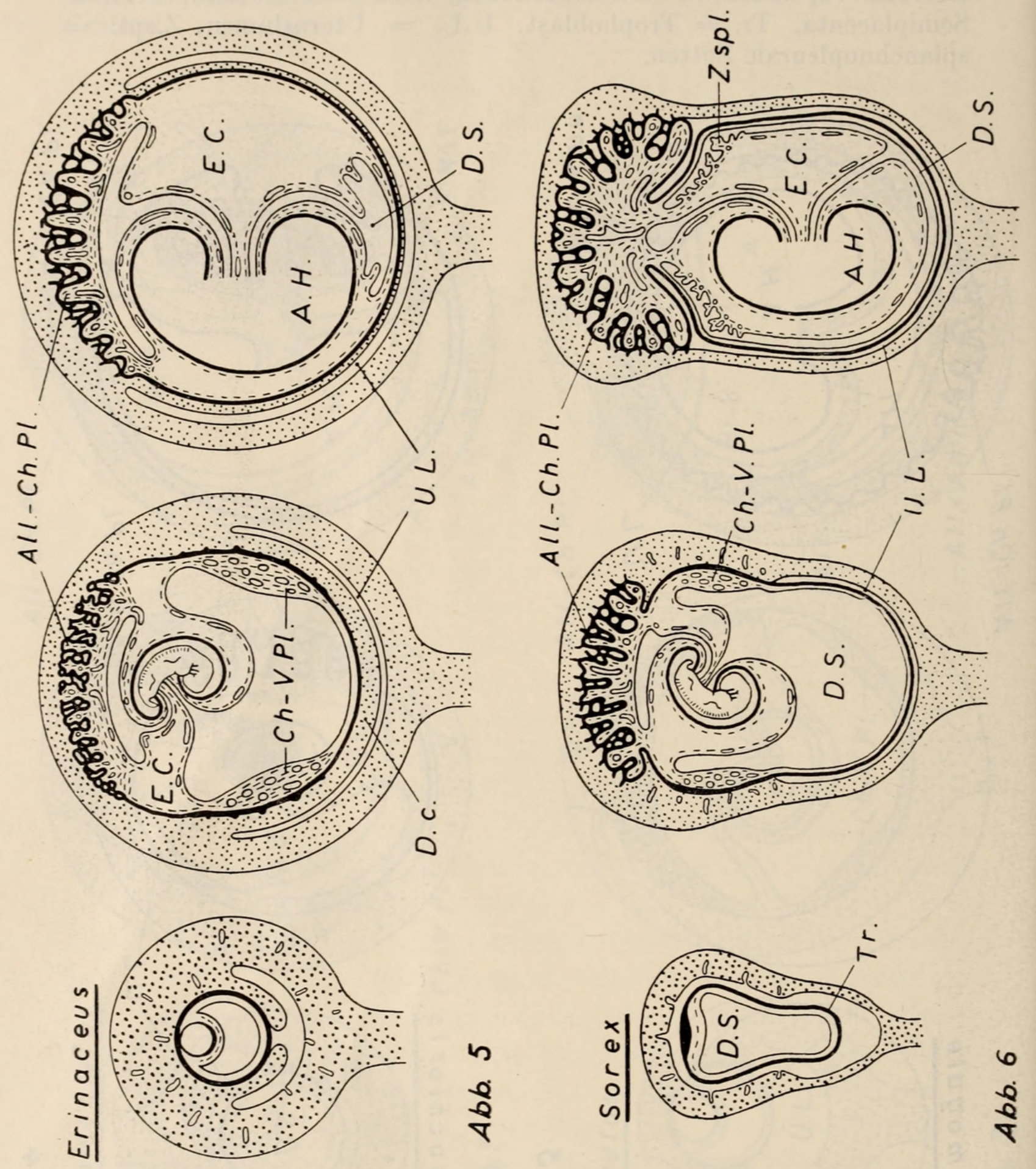


\section{$2 \mathrm{BHL}$ Biodiversity Heritage Library}

Strauss, F. 1942. "Vergleichende Beurteilung der Placentation bei den Insektivoren." Revue suisse de zoologie 49, 269-282.

https://doi.org/10.5962/bhl.part.117707.

View This Item Online: https://www.biodiversitylibrary.org/item/148538

DOI: https://doi.org/10.5962/bhl.part.117707

Permalink: https://www.biodiversitylibrary.org/partpdf/117707

\section{Holding Institution}

American Museum of Natural History Library

\section{Sponsored by}

BHL-SIL-FEDLINK

\section{Copyright \& Reuse}

Copyright Status: In copyright. Digitized with the permission of the rights holder.

Rights Holder: Muséum d'histoire naturelle - Ville de Genève

This document was created from content at the Biodiversity Heritage Library, the world's largest open access digital library for biodiversity literature and archives. Visit BHL at https://www.biodiversitylibrary.org. 